\title{
Chunking, sorting, and rule-learning from serial patterns of brain-stimulation reward by rats
}

\author{
STEPHEN B. FOUNTAIN and ZOLTAN ANNAU \\ Department of Environmental Health Sciences, The Johns Hopkins University, Baltimore, Maryland
}

\begin{abstract}
Three studies tested the notion that rats would treat brain-stimulation reward (BSR) as a stimulus alphabet from which rules could be abstracted to learn serial patterns. In Experiment 1, rats learned to track a serial pattern of 18-10-6-3-1-0 pulses of BSR, responding fast in anticipation of large quantities of BSR and slowly or not at all in anticipation of small quantities of BSR. In Experiment 2, rats learned to track a formally simple 18-6-1-0 pattern faster than a formally complex 18-1-6-0 pattern in a within-subjects procedure, indicating that rats can learn to discriminate between simple and complex pattern structures. Finally, in Experiment 3 rats learned either a formally simple 25-18-10-3-1-0 or a formally complex 25-3-10-18-1-0 pattern whose successive elements were separated by an embedded three-element 6-6-0 subpattern. Rats learned to "chunk together" the dispersed pattern elements, and rats receiving the simple pattern learned to track their pattern, whereas rats receiving the complex pattern did not. The latter results suggest that when simple pattern structure is available, rats can simultaneously track rule structures in at least two memory locations. The results of these experiments, using a new testing procedure and, presumably, a new stimulus alphabet, generalize and extend the idea that rats can abstract relational rules to learn serial patterns.
\end{abstract}

The rat's response to serial patterns composed of stimuli such as quantities of food has been viewed as resulting from one or more of several pattern-learning strategies. For example, rats appear to have a predilection to abstract and use general principles, or "rules," that describe or predict whole sets of pattern elements (i.e., the "rule-learning" view; Hulse, 1978; Hulse \& Dorsky, 1977, 1979). Another suggested strategy is memory of the last pattern element or last several pattern elements encountered as a cue or cues for anticipating successive pattern elements (i.e., the "memory-of-priorreward" and the "multiple hedonic memory" views of serial pattern learning; Capaldi, 1967; Capaldi \& Molina, 1979; Capaldi \& Verry, 1981). In another strategy. responses to one food quantity in a sequence can be modulated by the magnitude of the quantity immediately following it in the sequence. Moreover, in the case in which more than one sequence is presented in succession, the response to the terminal food quantity of one sequence can be modulated by the magnitude of the initial element of the sequence immediately following it (i.e., the "serial mapping" view; Capaldi, Nawrocki, \& Verry, 1983). Finally, rats are sensitive to extrapattern

\footnotetext{
This research was supported by National Institutes of Health Research Service Award MH 08759 from the National Institute of Mental Health to S. B. Fountain, and by ES 07094 and ES 02277 to Zoltan Annau. We thank David E. Schenk and Jeffrey L. Almony for their role in conducting surgery and in testing rats, and Robert E. Lintz for technical assistance. We also thank Maria S. Zaragoza for her comments on an earlier version of the article. Reprints may be obtained from Stephen B. Fountain, Department of Psychology, Kent State University, Kent, $\mathrm{OH} 44242$
}

cues that can facilitate or hinder their ability to "chunk" a pattern into meaningful groups of pattern elements from which rules may be abstracted easily (Fountain, Henne, \& Hulse, 1984). The conclusion to be drawn from this short inventory is that rats may employ a variety of strategies to use both intra- and extrapattern information in serial pattern learning.

One question of interest is the extent to which rats are able to marshal the various kinds of information available to them to learn patterns of greater and greater length, complexity, or organization, and what strategies prove to be most useful in this regard. When patterns are long but highly organized, what strategies can the rat utilize to learn the pattern? One highly organized kind of serial pattern that is difficult for humans is one that requires them to keep track of two or more sets of rules in two or more memory locations. An example of such a pattern would be 1-8-8-9-2-8-8-9-3-8-8-9-4-8-8-9-5. To learn this pattern, the most efficient strategy would be to "chunk together" pattern elements by first sorting elements into "chunks" and then learning rules that described the relationships of successive pattern elements within "chunks." The sorting process begins when the subject notices that an 8-8-9 subsequence recurs periodically, but that elements between subsequences do not recur periodically. Once the elements 1-2-3-4-5 are sorted into one memory location and the elements 8-8-9 are sorted into another, rules relating pattern elements within chunks can be abstracted and learned. In this case, all the elements of the 1-2-3-4-5 chunk are related by a single " +1 " rule; learning the single rule describing the chunk is a more efficient strategy than learning a series of interitem associations by rote. By using this 
strategy, humans are able to learn this kind of pattern relatively quickly and easily because they can learn relational rules between elements that may be widely dispersed within a serial pattern, though this type of pattern is one of the more difficult for humans to learn (Simon \& Kotovsky, 1963).

Studies have already shown that rats have a predilection to use "chunking" and rule-learning to learn long, well-organized patterns (Fountain et al., 1984). If rats have the capacity to sort pattern elements into appropriate chunks held in different memory locations, rulelearning combined with these chunking and sorting processes would be the most efficient method of learning patterns similar to the one described above. Of course, rats could use many other kinds of strategies-for example, memory-of-prior-reward, multiple hedonic memory, serial mapping, or others - to learn this kind of long and difficult serial pattern. Experiment 3 examined rats' strategy in serial learning for patterns of this type. Because of the length and difficulty of this kind of pattern, a more efficient operant method was needed for studying serial pattern learning in rats. The development of this method is reported in Experiments 1 and 2 .

The methodological difficulty that arises when patterns must be composed of many food quantities, and of necessity some quantities must be quite large, is that fewer repetitions of a pattern may be presented each day before the rats become satiated. For example, Fountain et al. (1984) were able to present their rats only one 25-quantity pattern each day. If the pattern also happens to be very difficult for rats to learn (perhaps aggravated by the 23-h interpattern interval), the time required to present rats a sufficient number of pattern repetitions to produce learning may be prohibitive. To overcome this problem, brain-stimulation reward (BSR) quantity was chosen as a stimulus dimension from which pattern elements could be drawn to create serial patterns. One advantage of using BSR quantities in serial patterns is that rats may receive many patterns within a daily session without satiation. A second advantage of using serial patterns of BSR is that the experimenter has precise control of the onset and offset of reward administration; operant serial learning methods using food quantity stimuli (e.g., Fountain, Evensen, \& Hulse, 1983, Experiment 2) lack strict control of food consumption time, and thus lack strict control of intertrial interval. Finally, it might be argued that food quantity and BSR quantity have basic similarities (Olds, 1956) that would facilitate comparisons between methods.

In Experiment 1, rats received an 18-10-6-3-1-0 pattern of BSR quantities for Days 1 and 2. Quantities were delivered on successive trials for leverpresses in a discrete-trial operant procedure. Response latency was used as an index of quantity anticipation. We predicted that if rats perceived BSR quantity as a stimulus dimension with ordinal properties, they would learn to track (Hulse, 1978; Restle \& Burnside, 1972) their serial pat- tern by responding fast for large quantities and slowly, or not at all, for small quantities of BSR. Because we found that rats responded more slowly for the initial 18-pulse quantity of BSR than for the 10-pulse quantity of BSR that followed on the next trial, the pattern was changed to 25-18-6-3-1-0 on Day 3. This change of procedure was used to determine whether rats simply failed to anticipate the end of the relatively long interpattern interval, and thus produced a slightly longer latency for the 18-pulse quantity, or that the 18-pulse BSR quantity was so much stimulation that it was aversive (cf. Stein, 1962).

\section{EXPERIMENT 1}

\section{Method}

Subjects. The subjects were two naive male hooded rats obtained from Blue Spruce Farms, Altamont, New York. They weighed 300 $350 \mathrm{~g}$ at the time of surgical implantation of electrodes and were approximately $80-100$ days of age at the beginning of the experiment. The rats were housed in individual plastic tubs, and were provided free access to food and water in their home cages throughout the experiment

Apparatus. Test chambers $(30 \times 30 \times 30 \mathrm{~cm})$ were composed of clear Plexiglas walls and a floor of stainless steel rods $(0.5 \mathrm{~cm}$ in diameter) spaced $1.25 \mathrm{~cm}$ apart. One end wall was equipped with a response lever located $4.0 \mathrm{~cm}$ from the front wall and $5.0 \mathrm{~cm}$ above the floor. In the testing chamber, the rats were connected to a stimulator by way of a flexible cord (Plastic Products MS304) and a commutating device that allowed the animal free movement within the chamber. The operant chamber was enclosed in a sound-attenuating hull $(55 \times 50 \times 55 \mathrm{~cm})$ made of particle board. A houselight $(7.5 \mathrm{~W})$ was centered approximately $5 \mathrm{~cm}$ below the ceiling on the rear wall of the hull. The experiment was controlled from an adjoining room by a Digital Equipment Corporation PDP-8/e minicomputer and SKED software system (State Systems, Inc., Kalamazoo, Michigan).

Procedure. Each rat was anesthetized by pentobarbitol injection. A twisted stainless steel bipolar electrode (Plastic Products MS303/1) was then implanted unilaterally in the posterior lateral hypothalamus of each rat. With the skull level, the coordinates were $4.5 \mathrm{~mm}$ posterior to the bregma, $1.5 \mathrm{~mm}$ lateral to the midline, and $8.5 \mathrm{~mm}$ below the surface of the skull.

The rats were allowed 5-7 days for recovery from surgery, then they were shaped to leverpress for brain-stimulation reward (BSR). A $60-\mathrm{Hz}$ sinusoidal pulse train of $100 \mathrm{msec}$ duration from a constant current source of 25-40 $\mu \mathrm{A}$ was delivered for shaping and for each leverpress. The rats received 1 day of shaping followed by 3 days of continuous reinforcement training, in which each leverpress was followed by one 100-msec pulse of BSR. These daily sessions lasted $1 \mathrm{~h}$. Current was adjusted for each rat to produce 5,000-10,000 leverpresses per hour. Rats that failed to produce at least 5,000 leverpresses on the last day of training were excluded from the experiment.

For 2 days prior to the beginning of the experiment proper, the rats received further leverpress training in a procedure designed to prepare them for the discrete-trial procedure of the testing phase. For $1 \mathrm{~h}$ each day, the rats were allowed to leverpress in the operant chamber. Throughout the session, the houselight was first turned on for $2 \mathrm{~min}$, then off for $15 \mathrm{sec}$. Each leverpress was rewarded by a 100-msec pulse of BSR when the houselight was on, but leverpresses were never rewarded when the houselight was off.

On Days 1-2 of the experiment proper, each rat was placed in an operant chamber with the houselight off. Each trial began when the houselight was turned on and continued until the rat leverpressed or until $30 \mathrm{sec}$ elapsed. Immediately following delivery of BSR or termination of the trial, the houselight was extinguished. On successive 
trials. leverpresses while the light was on produced tirst 18 pulses of BSR, then 10 pulses of BSR, then 6, 3, I and tinally 0 pulses of BSR. BSR quantities were 100 msec pulses of stimulation separated by $100 \mathrm{msec}$. No BSR was delivered on trials in which rats failed to leverpress. The houselight was extinguished between trials and between patterns. Trials that made up the 18 10-6-3-1-0 pattern were separated by a 1 -sec intertrial interval. Patterns were separated by a 15 -sec interval. Each rat received 100 repetitions of the pattern each day. Following testing, the rats were removed from the operant chamber and returned to their home cages. A response latency, the amount of time elapsing between the beginning of the trial and the first leverpress, was recorded to the nearest $0.01 \mathrm{sec}$ for each trial. A 30 -sec response latency was recorded for trials in which no leverpress occurred.

On Day 3, the pattern was changed from 18-10-6-3-1-0 to 25-18-6-3-1-0. All other procedures were identical to those used on Days $1-2$.

\section{Results}

Figure 1 shows the two rats' response to the patterns they learned in Experiment 1. Each rat's mean response latency is shown for each quantity of the pattern for blocks of 10 pattern repetitions at selected points during acquisition. Block I (B1) was composed of repetitions 1-10, Block 5 (B5) was composed of repetitions 41-50, and Block $10(\mathrm{~B} 10)$ was composed of repetitions 91-100 (the last block of repetitions for Day 1). Similarly, Block 20 (B20) was composed of repetitions 191-200,

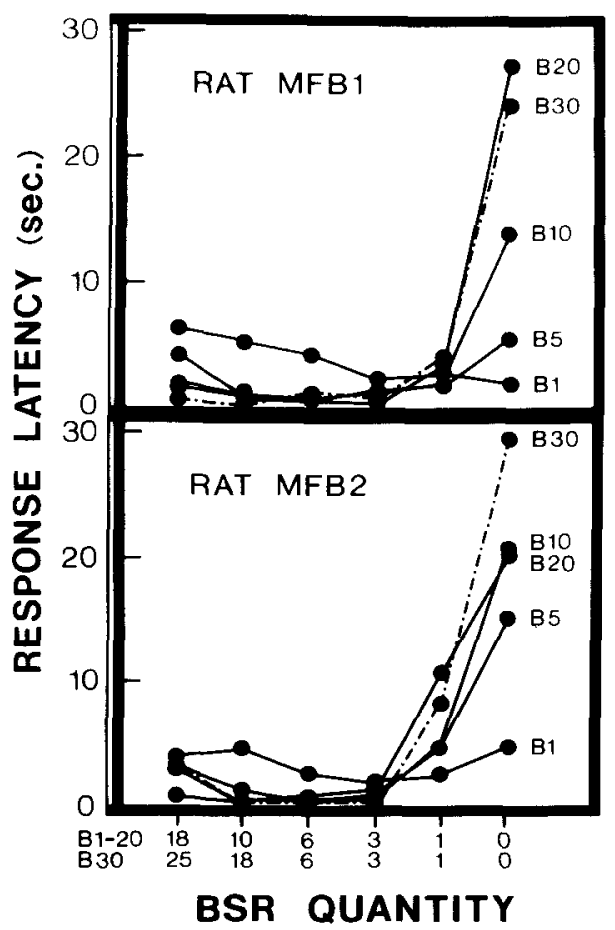

Figure 1. Each of two rats' mean response latencies for BSR quantities that composed their serial patterns for blocks of 10 pattern repetitions at selected points during acquisition. Block 1 (B1) was composed of pattern repetitions 1-10, Block 5 (B5) of repetitions 41-50, Block 10 (B10) of repetitions 91-100 (the last block of Day 1), Block 20 (B20) of repetitions 191-200 (the last block of Day 2), and Block 30 (B30) of repetitions 291-300 (the last block of Day 3). The rats received an 18-10-6-3-1-0 pattern of BSR quantities for Blocks 1-20 (i.e., Days 1 and 2, solid lines) and a 25-18-6-3-1-0 pattern for Blocks 21-30 (Day 3, dashed lines). the last block of repetitions for Day 2, and Block 30 (B30) was made up of repetitions 291-300, the last block of repetitions for Day 3. The rats received the pattern 18-10-6-3-1-0 on Days 1 and 2 and 25-18-6-3-1-0 on Day 3 . The rats showed remarkably similar acquisition of pattern tracking. Both rats learned first to slow their responses in anticipation of the 0-pulse BSR quantity, then later showed a tendency to respond more slowly in anticipation of the 1-pulse quantity than they did in anticipation of larger quantities. Planned comparisons on means for the two rats showed that they responded reliably more slowly for the 0-pulse quantity than they did for other quantities by Block $10(\mathrm{p}<.05)$ and continued to do so throughout the remainder of the experiment $(p<$ $.05)$. The rats also responded more slowly for the 1pulse quantity than they did for larger quantities on Block $20(p<.05)$. Furthermore, both rats showed a tendency to respond slightly (but not significantly) more slowly on 18-pulse trials that initiated a pattern than they did on the 10-pulse trial that followed it, but when the 18-pulse quantity was moved to the second position of the pattern on Day 3, both rats responded very quickly in anticipation of it. Also, increasing the initial BSR quantity from 18 to 25 pulses produced no decrease in response latency on the first trial of the pattern $(p>$ $.05)$.

The planned comparisons reported above were computed using the appropriate error term from an analysis of variance (ANOVA) of the raw data represented by the means shown in Figure 1. The analysis revealed significant main effects for blocks $[F(4,4)=6.86, p<.05]$ and quantities $[F(5,5)=36.10, p<.01]$ and a significant interaction of blocks $\times$ quantities $[F(20,20)=7.20, p<$ $.01]$.

\section{Discussion}

Rats in Experiment 1 learned to respond fast for large quantities of BSR and slowly, or not at all, for small quantities of BSR. One explanation for these results might be that the rats simply counted trials or used temporal anticipation as a strategy for anticipating small quantities of BSR. Because rats have difficulty counting or using a temporal anticipation strategy in other serial learning tasks (Hulse \& Campbell, 1975), a better explanation is that the rats were able to discriminate the BSR quantities of their serial pattern, and that they perceived BSR quantity as a stimulus alphabet (Hulse \& O'Leary, 1982; Jones, 1974), a set of stimuli drawn from a stimulus dimension having at least ordinal properties. Stimuli composing a stimulus alphabet have the unique characteristic that they can be related to each other by quantitative descriptions such as "greater than," "less than," " +1 ," " -1 ," and so on. These quantitative descriptions are one kind of rule that can be used to describe relationships among elements of a serial pattern. Rules describing a pattern are a source of information that can sometimes be used to facilitate pattern learning and memory. The results of Experiment 1 suggest that BSR quantity is a stimulus dimension that rats 
may be able to use in a relational manner to learn serial patterns. Experiment 2 tested rats' predilection to use a rule-learning strategy to learn serial patterns composed of BSR quantities.

\section{EXPERIMENT 2}

The rule-learning hypothesis assumes that rats actively search patterns for formally simple features of pattern structure and that they learn rules to facilitate learning the simple structure they find. To test the hypothesis that rats would use a rule-learning strategy to learn serial patterns composed of BSR quantities, each rat in Experiment 2 received two serial patterns that alternated throughout each daily session of 100 patterns. One pattern, an 18-6-1-0 pattern of BSR quantities, was monotonically decreasing, that is, the successive elements of the pattern were always "less than" preceding quantities. The second pattern, an 18-1-6-0 pattern, was nonmonotonically decreasing. The nonmonotonic pattern is described by more than one rule, both "less than" and "greater than" rules. The monotonic 18-6-1-0 pattern is said to be formally simple because the relationships between all pairs of successive pattern elements can be described by a single rule. The nonmonotonic 18-6-1-0 pattern is formally more complex because more rules are required for an adequate description of the pattern. Under appropriate conditions (Hulse, 1980; Roitblat, Pologe, \& Scopatz, 1983), pattern complexity is a good predictor of pattern difficulty (Fountain et al., 1983; Fountain \& Hulse, 1981; Hulse \& Dorsky, 1977); apparently, learning a pattern described by a single rule is easier than learning a pattern described by many rules. Learning a single rule to describe a pattern is also more efficient than learning the same pattern using other strategies that require remembering individual elements and their positions in the pattern. Although rats could adopt other strategies for learning their patterns, we predicted that the rats would adopt the more efficient rule-learning strategy and thus would learn to track their monotonic pattern faster and better than their nonmonotonic pattern.

\footnotetext{
Method

Subjects. The subjects were four naive male hooded rats obtained from Blue Spruce Farms, Altamont, New York. The rats weighed $300-350 \mathrm{~g}$ at the time of surgery and were approximately $80-100$ days of age at the beginning of the experiment. The rats were housed in individual plastic tubs, and were provided free access to food and water in their home cages.

Apparatus. The apparatus was the same as that used in Experiment 1 .

Procedure. The procedures used for implantation of electrodes and for all leverpress training prior to the experiment proper were identical to those used in Experiment 1. The procedure for testing rats during the experiment proper was identical to that used in the first experiment with the exceptions that follow. The rats received two patterns instead of one; they received a monotonic 18-6-1-0 pattern for the first pattern of each day and a nonmonotonic 18-1-6-0 pattern for the second pattern. Thereafter the two patterns alternated throughout the daily session. Pattern quantities were presented following leverpresses in the
}

discrete trial procedure used in Experiment 1, but trials lasted $15 \mathrm{sec}$ instead of $30 \mathrm{sec}$. Trials that made up patterns were separated by a 10 -sec intertrial interval, and patterns were separated by $1 \mathrm{~min}$. As before, the houselight was extinguished during the intervals between trials and between patterns. The rats received 50 monotonic and 50 nonmonotonic patterns each day for 7 days.

\section{Results}

The results of Experiment 2 are most clearly summarized by comparing acquisition of tracking performance for 1- and 0-pulse BSR quantities. These results are shown in Figure 2. Mean response latencies for the four rats are shown for monotonic and nonmonotonic 1- and 0 -pulse trials for each day of the experiment. Overall, the rats responded more slowly on monotonic 1- and 0 -pulse trials than on nonmonotonic 1 - and 0 -pulse trials, and planned comparisons showed that the rats first responded reliably more slowly on 1- and 0 -pulse trials in monotonic than in nonmonotonic patterns on Day 4 $(p<.05)$. Similarly, the rats responded reliably more slowly on 0-pulse trials than on 1-pulse trials, and this difference also first became significant on Day 4 $(p<.05)$. These planned comparisons were based on an ANOVA performed on means for 1 - and 0 -pulse trials for blocks of 10 patterns for each day of the experiment. Thus, the analysis included patterns, quantities, days, and blocks as factors. The analysis revealed reliable main effects for patterns $[F(1,3)=80.88, p<.01]$ and for quantities $[F(1,3)=39.17, p<.01]$. Significant interactions included days $\times$ patterns $[F(6,18)=5.72, p<$ $.01]$ and days $\times$ quantities $[\mathrm{F}(6,18)=15.93, \mathrm{p}<.01]$. No other reliable effects were indicated $(p>.05)$.

A similar ANOVA was conducted to compare anticipatory responding for the 0 -pulse quantity alone of monotonic and nonmonotonic patterns over the entire experiment, with each day represented by means for blocks of 10 patterns. The analysis revealed reliable

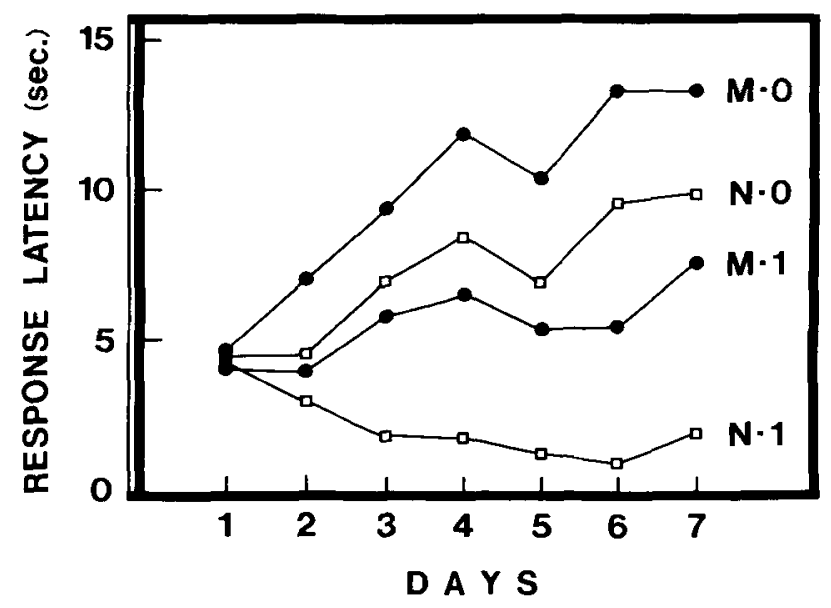

Figure 2. Mean response latencies for 1- and 0-pulse trials of monotonic (M) and nonmonotonic $(N)$ patterns for each of 7 days. Each rat received both the monotonic 18-6-1-0 pattern and the nonmonotonic 18-1-6-0 pattern. 
main effects for days $[F(6,18)=6.79, p<.01]$ and for patterns $[F(1,3)=10.26, p<.05]$. No other significant effects were detected $(p>.05)$. The results of this analysis support the contention that rats responded more slowly in anticipation of the 0-pulse BSR quantity of the monotonic pattern than for the 0-pulse quantity of the nonmonotonic pattern over the course of the experiment.

A further analysis was conducted to assess pattern tracking on Day 7 , the last day of the experiment. Planned comparisons showed that the rats responded reliably more slowly on 1 - and 0 -pulse trials than on 18and 6-pulse trials of the monotonic pattern, but that they responded reliably more slowly only on 0-pulse trials relative to other quantities for the nonmonotonic pattern $(\mathrm{ps}<.05)$. In fact, the rats' mean response latency was shorter for the 1-pulse trial of the nonmonotonic pattern than for 18- and 6-pulse trials, though these differences were not reliable $(p>.05)$. Planned comparisons were computed using the appropriate error term from an ANOVA conducted on the rats' mean response latencies for all quantities by blocks of 10 patterns for Day 7 . The analysis revealed significant main effects for patterns $[F(1,3)=28.36, p<.05]$ and for quantities $[F(3,9)=36.24, p<.01]$. Significant interactions included blocks $\times$ quantities $[\mathrm{F}(12,36)=2.36$, $\mathrm{p}<.05]$ and patterns $\times$ quantities $[\mathrm{F}(3,9)=6.56, \mathrm{p}<$ $.05]$.

\section{Discussion}

Rats in Experiment 2 learned to discriminate monotonic and nonmonotonic patterns, and learned to track their monotonic 18-6-1-0 pattern faster and better than their nonmonotonic 18-1-6-0 pattern. By the end of the experiment, the rats responded reliably more slowly for 1 - and 0-pulse quantities of the monotonic pattern than for larger quantities, but they responded reliably more slowly only to 0-pulse quantities of the nonmonotonic pattern than to other quantities. In addition, better pattern tracking was reflected in longer response latencies for 1 - and 0 -pulse quantities of the monotonic pattern than for 1- and 0-pulse quantities of the nonmonotonic pattern throughout the experiment.

The results of Experiment 2 support the rule-learning view of rat serial pattern learning. This view can be summarized as follows: (1) Rats discriminated the BSR quantities that made up their serial patterns; (2) they perceived BSR quantities as elements of an ordered stimulus alphabet (Hulse \& O'Leary, 1982; Jones, 1974); (3) they abstracted rules relating successive BSR quantities as the quantities were encountered; and (4) when a rule appeared consistently, they remembered some representation (Roitblat, 1982) of the rule as a summarization of the relationships among a whole set of quantities. Better anticipation of the 0-pulse quantity of the monotonic than of the nonmonotonic pattern occurred because the relationships between successive BSR quantities that composed the monotonic pattern could be described by fewer rules than those of the nonmonotonic pattern; again, learning one rule to describe a serial pattern was easier than learning more rules.

\section{EXPERIMENT 3}

The results of Experiment 2 support the idea that rats are able to discriminate patterns that occur within a daily testing session. Experiment 3 tested the idea that rats have the capacity to discriminate pattern structures within patterns, and that they can hold in memory some representation of one structural chunk while responding to the elements of a second structural chunk of a pattern. To test this notion, rats received either a monotonically decreasing 25-18-10-3-1-0 pattern or a nonmonotonic 25-3-10-18-1-0 pattern of BSR quantities. In each case, however, the BSR quantities were separated by embedded 6-6-0 subpatterns. Differential anticipation of the 1 - and 0 -pulse quantities by monotonic and nonmonotonic groups required that the rats remember the occurrence of pattern elements many trials earlier. Differential response to the 1 -pulse quantity relative to larger quantities required the memory of the 3- or 18pulse quantity (for monotonic and nonmonotonic patterns, respectively) four trials earlier, because the 6-6-0 subpattern immediately preceding the 1-pulse quantity also preceded 18-, 10-, 3-, and 0-pulse quantities. Thus, remembering 6-6-0 immediately prior to the 1-pulse trial would not be sufficient as a discriminative cue. Similar logic shows that differential anticipation of the 0-pulse quantity relative to larger quantities should be at least as difficult as predicting the 1-pulse quantity. Furthermore, differential anticipation of the terminal 0-pulse quantity between groups requires that rats use information about pattern elements occurring at least eight trials earlier. Differential anticipation of 1- and 0-pulse quantities between groups would indicate that rats can learn to respond to the sequential order of stimuli widely separated in a pattern. Specifically, if rats could "chunk together" the widely dispersed elements of the monotonic or nonmonotonic pattern, rule-learning would be possible within the 25-18-10-3-1-0 chunk, but not within the 25-3-10-18-1-0 chunk. Differential anticipation of small and large BSR quantities should develop more rapidly for the monotonic than for the nonmonotonic pattern; learning a single "less than" rule to relate the successive elements of the monotonic pattern should be relatively more efficient and easier than learning many rules and their order to describe the more complex nonmonotonic pattern. Of course, rats could adopt other strategies to learn this complex pattern, with or without the use of "chunking" processes, but we predicted that they would adopt the easier and more efficient rule-learning strategy.

\footnotetext{
Method

Subjects. The subjects were 18 naive male hooded rats obtained from Blue Spruce Farms, Altamont, New York. They weighed 300 -
} 
$350 \mathrm{~g}$ at the time of surgery and were approximately 80-100 days of age at the beginning of the experiment. The rats were housed in individual plastic tubs, and were provided free access to food and water in their home cages.

Apparatus. The apparatus was the same as that used in Experiments 1 and 2 .

Procedure. The procedures used for implantation of electrodes and for all leverpress training prior to the experiment proper were identical to those used in Experiments 1 and 2. The general procedure for testing rats during the experiment proper was identical to that used in Experiment $\mathbf{l}$ with the following exceptions. The rats were assigned randomly to the two pattern conditions so that nine rats received a monotonic 25-18-10-3-1-0 pattern of BSR quantities and nine received a nonmonotonic 25-3-10-18-1-0 pattern. In addition, a 6-6-0 subpattern of BSR quantities occurred between every pair of quantities composing monotonic or nonmonotonic patterns. The resulting dispersed monotonic pattern with its embedded 6-6-0 subpatterns was

25 6-6-0 18 6-6-0 10 6-6-0 3 6-6-0 1 6-6-0 0,

and the dispersed nonmonotonic pattern with its embedded 6-6-0 subpatterns was

\section{6-6-0 3 6-6-0 10 6-6-0 18 6-6-0 1 6-6-0 0.}

Pattern quantities were presented following leverpresses in the discrete trial procedure used in Experiment 1 , in which trials lasted a maximum of $30 \mathrm{sec}$. Trials that made up 6-6-0 subpatterns were separated by a 1 -sec intertrial interval (represented by the dashes in the patterns above). Embedded 6-6-0 subpatterns were separated from quantities of dispersed monotonic and nonmonotonic patterns by 5 -sec intervals (represented by spaces in the patterns above). Pattern repetitions were separated by $2 \mathrm{~min}$. As before, the houselight was extinguished during the intervals between trials and between patterns. Rats received 10 repetitions of either the dispersed monotonic or the dispersed nonmonotonic pattern each day for 14 days.

\section{Results}

The data for the dispersed monotonic and nonmonotonic pattern elements were analyzed separately from the data for the embedded 6-6-0 subpatterns. Planned comparisons for the dispersed patterns alone revealed that rats in the dispersed monotonic (M) condition learned to track their 25-18-10-3-1-0 pattern whereas rats in the dispersed nonmonotonic $(\mathrm{N})$ condition never learned to track their 25-3-10-18-1-0 pattern. Rats in the M condition responded reliably more slowly for both the 1 - and the 0-pulse quantities than for the 25-, 18-, and 10-pulse quantities on Days $8,10,11,12,13$, and 14 (ps $<.05$ ). Rats in the $\mathrm{N}$ condition never differentiated their response latencies in anticipation of any elements of their 25-3-18-10-1-0 pattern (ps $>.05$ ). Furthermore, rats in $M$ responded reliably more slowly in anticipation of their 1- and 0-pulse BSR quantities than did rats in $\mathrm{N}$ on Days $10-14$ (ps $<.05$ ). Figure 3 shows the results for both dispersed and embedded patterns for Days 12-14, the last 3 days of the experiment. Rats in $M$ responded reliably more slowly for 1- and 0-pulse BSR quantities of their dispersed 25-18-10-3-1-0 pattern than for larger quantities of their dispersed pattern; they also responded more slowly for 1 and 0 of their dispersed 25-18-10-3$1-0$ pattern than did rats in $\mathrm{N}$ for any quantity of their dispersed 25-3-10-18-1-0 pattern (ps $<.05$ ).

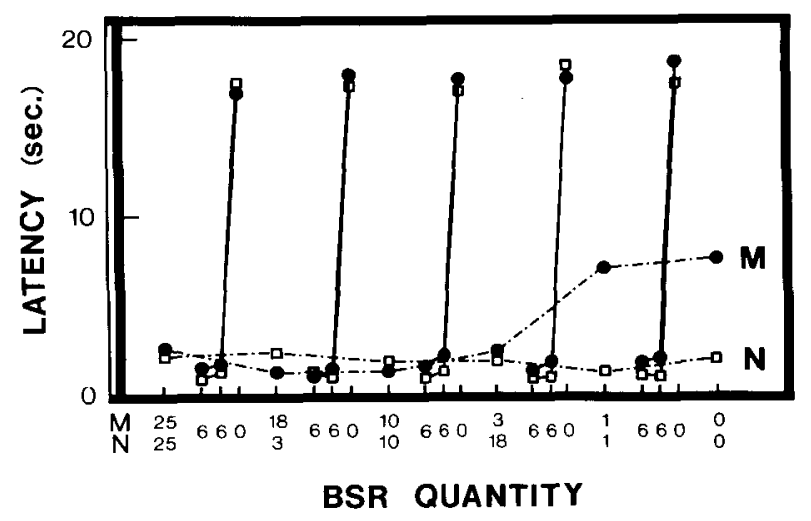

Figure 3. Mean response latencies for each BSR quantity that composed dispersed monotonic (M) and dispersed nonmonotonic (N) patterns (dashed lines), and their respective embedded patterns (solid lines), for Days 12-14, the last 3 days of the experiment. The dispersed monotonic pattern was 25-18-10-3-1-0, and the dispersed nonmonotonic pattern was 25-3-10-18-1-0. The quantities of both patterns were always separated by an embedded 6-6-0 subpattern.

The planned comparisons just reported were based on the appropriate error term from an ANOVA conducted on rats' daily mean response latencies for each quantity of their dispersed patterns; data for the embedded 6-6-0 subpatterns were not included in the analysis. The ANOVA revealed a significant main effect for quantities $[F(5,80)=18.39, p<.01]$, and significant interactions for groups $\times$ quantities $[\mathrm{F}(5,80)=23.24, \mathrm{p}<.01]$, days $\times$ quantities $[\mathrm{F}(65,1054)=4.08, \mathrm{p}<.01]$, and groups $\times$ days $\times$ quantities $[\mathrm{F}(65,1054)=2.97, \mathrm{p}<$ .01]. No other significant effects were indicated $(p>$ $.05)$.

Figure 3 also shows results for the embedded 6-6-0 patterns for Days 12-14, the last 3 days of the experiment. The results for embedded 6-6-0 subpatterns are shown in their proper location relative to elements of either the dispersed monotonic or the dispersed nonmonotonic pattern. Planned comparisons for the embedded 6-6-0 subpatterns showed that rats in both $M$ and $N$ learned to respond reliably more slowly for the 0-pulse BSR quantity than for the two 6-pulse quantities for Days 5-14 (ps <.05), but that rats in $M$ and $N$ never differed reliably in this regard $(p>.05)$. Planned comparisons failed to indicate reliable differences between $M$ and $\mathbf{N}$ groups at any time during the experiment (ps > .05 ), although an ANOVA computed on daily mean response times for the elements of each of the five 6-6-0 subpatterns for each day of the experiment indicated one reliable interaction involving groups as a factor. The ANOVA revealed significant main effects for days $[\mathrm{F}(13,208)=7.77, \mathrm{p}<.01]$, subpattern location $[\mathrm{F}(4,64)$ $=4.69, \mathrm{p}<.01]$, and quantities $[\mathrm{F}(2,32)=94.00, \mathrm{p}<$ $.01]$. Significant interactions included groups $\times$ subpattern location $[F(4,64)=6.11, p<.01]$, days $\times$ quantities $[\mathrm{F}(26,416)=37.43, \mathrm{p}<.01]$, and subpattern location $\times$ 
quantities $[F(8,128)=7.08, p<.01]$. No other significant effects were indicated (ps $>.05$ ). The significant groups $\times$ subpattern location interaction was not the result of anticipation of future BSR quantities. Rats in M responded more slowly on each successive 6-6-0 subpattern (when data were pooled for the three pattern elements), producing mean response latencies of 4.88 , $4.88,5.41,5.63$, and $5.68 \mathrm{sec}$ for the five successive 6-6-0 subpatterns, respectively. Rats in $\mathrm{N}$ showed no systematic pattern of response. Their mean response latencies were $5.34(3), 5.65(10), 5.59(18), 5.49(1)$, and 5.34 (0) sec for the five successive 6-6-0 subpatterns, respectively, where the quantity in parenthesis following each mean response latency indicates the BSR quantity that followed on the next trial. Rats in $\mathrm{N}$ did not respond more slowly on each successive 6-6-0 subpattern, nor did they order their responses by responding more slowly for subpatterns preceding small quantities of their dispersed nonmonotonic pattern and faster for subpatterns preceding larger quantities. In fact, the opposite trend is more apparent. However, as indicated above, none of the differences among these means are significant by planned comparisons (ps $>.05$ ).

To assess differences in acquisition of pattern tracking for embedded versus dispersed patterns, planned comparisons were conducted comparing response latencies for the 0-pulse BSR quantities of embedded and dispersed patterns. These planned comparisons showed that rats in $\mathrm{M}$ and $\mathrm{N}$ responded reliably more slowly for the 0 -pulse quantities of the embedded patterns than for the 0 quantity of the dispersed patterns for Days 5-14 (ps < .05 ). This analysis also showed that rats in $M$ responded reliably more slowly for the 0 -pulse quantity of their dispersed pattern than rats in $\mathrm{N}$ did for the 0 -pulse quantity of their dispersed pattern for Days 11-14 (ps<.05). These planned comparisons were based on the appropriate error term of an ANOVA conducted on daily mean response latencies of 0 -pulse trials of both embedded and dispersed patterns for the 14 days of the experiment. The ANOVA revealed significant main effects for days $[F(13,208)=26.93, p<.01]$ and for location (of the 0 pulse quantity) $[F(5,80)=64.00, p<.01]$. Significant interactions included groups $\times$ location $[F(5,80)=2.58$, $\mathrm{p}<.05]$, days $\times$ location $[\mathrm{F}(65,1040)=10.33, \mathrm{p}<.01]$, and groups $\times$ days $\times$ location $[\mathrm{F}(65,1040)=1.37, \mathrm{p}<$ $.05]$.

\section{Discussion}

Rats in the dispersed monotonic (M) condition of Experiment 3 learned to track their dispersed 25-18-10-3$1-0$ pattern, whereas rats in the dispersed nonmonotonic (N) condition never learned to track their dispersed 25-3-10-18-1-0 pattern. Pattern tracking for $M$ rats was reflected in reliably slower responses in anticipation of 1- and 0-pulse BSR quantities than for larger quantities. Serial pattern learning was thus shown to be a function of pattern complexity, despite the fact that elements that composed the patterns were separated by 6-6-0 subpat- terns. The results support the hypothesis that rats are able to sort into "chunks" pattern elements that were maintained in separate memory locations and that, once sorting is accomplished, rats in $\mathbf{M}$ are able to encode some representation of the single "less than" rule that relates successive elements of their formally simple dispersed 25-18-10-3-1-0 pattern. In contrast, rats in $\mathrm{N}$ never tracked their 25-3-10-18-1-0 pattern because no single rule described the pattern. Once again, learning a single rule was easier than learning several rules encountered in haphazard order. However, although rats in $\mathrm{N}$ never learned to track their complex 25-3-10-181-0 dispersed pattern, apparently they were able to sort pattern elements into appropriate chunks just as well as rats in $\mathrm{M}$ did; rats in $\mathrm{N}$ were as proficient at tracking their 6-6-0 subpatterns as were rats in $M$.

\section{GENERAL DISCUSSION}

A restatement of the rule-learning view of rat serial pattern learning is in order in view of the results of Experiment 3 , with the addition of some further specifications warranted by these results. First, rats demonstrated in each experiment that they discriminated the BSR quantities that made up their serial patterns. Discrimination was reflected in differential anticipatory responding for large and small quantities of BSR within patterns. Second, rats perceived the BSR quantities that made up patterns as elements drawn from a stimulus alphabet (Hulse \& O'Leary, 1982; Jones, 1974). Third, rats abstracted rules relating successive BSR quantities as the quantities were encountered. Fourth, when a rule appeared consistently, it was remembered as a summarization of the relationships among many quantities. If a single rule described all relationships among successive elements of a pattern, rule-learning was relatively easy, producing rapid acquisition of pattern tracking behavior. When many rules were required to describe a pattern, and thus each rule described relationships among fewer quantities, rule-learning was more difficult and acquisition of pattern tracking behavior was slower. The results of Experiments 2 and 3 indicate that rats can discriminate pattern structures. In addition, the results of Experiment 3 support the hypothesis that rats are able to use rules to describe pattern elements that are widely dispersed in a pattern by sorting elements of the same pattern into different structural "chunks" maintained in different memory locations.

Evidence for "chunking" processes (Bower \& Winzenz, 1969; Miller, 1956; Simon, 1974) in serial pattern learning has already been demonstrated in animals (Fountain et al., 1984; Pribram \& Tubbs, 1967; Tubbs, 1969). For example, rats learned a 25-element serial pattern made up of five repetitions of a 14-7-3-1-0 (pellets of 45-mg Noyes food pellets) subsequence, but had difficulty without the aid of "phrasing cues" (Fountain et al., 1984; Restle, 1972). Spatial cues that marked the boundaries of the 14-7-3-1-0 sub- 
sequence ("good" phrasing cues) facilitated learning over a control pattern that had no such cues, and spatial cues that did not correspond to subsequence boundaries ("bad" " phrasing cues) produced the poorest learning of all. Presumably, "good" phrasing facilitated the process of rule-learning by emphasizing boundaries between formally simple 14-7-3-1-0 "chunks" of the pattern, that is, sets of pattern elements related by a single rule. "Bad" phrasing, on the other hand, tended to disrupt the process of "chunking" pattern elements into appropriate formally simple groups. Experiments 2 and 3 demonstrated rats' predilection to use (and perhaps their dependence on) a rule-learning strategy and "chunking" processes in more demanding serial patternlearning tasks. The results of Experiments 2 and 3 suggest that rats' "chunking" abilities are extensive, and the results of Experiment 3 suggest that these abilities include processes for sorting sequential elements of a pattern into sets defined by characteristics unrelated to sequential proximity.

\section{Multiple Item Memory and Serial Mapping}

The results of Experiment 2 from a "two-series" (Capaldi \& Verry, 1981) or "concurrent-sequence" (Self \& Gaffan, 1983) procedure, in which rats learned to track a monotonic 18-6-1-0 pattern faster than a nonmonotonic 18-1-6-0 pattern, replicate results produced using single-series procedures (cf. Fountain et al., 1983; Hulse, 1978; Hulse \& Dorsky, 1977, 1979). Some researchers have suggested that rats use multipleitem memory only when other strategies fail, that is, when the memory of one event is not a sufficient cue for accurate anticipation. However, the results of singleseries procedures, in which multiple-item memory is not required to learn the patterns, and the two-series results of Experiment 2 are so similar that it seems unlikely that rats employed different strategies for the two procedures. Instead, multiple-item memory may be the strategy of choice, rather than of "last resort" (Capaldi \& Verry, 1981; Self \& Gaffan, 1983), when it provides a method of reducing memory load (Hulse, 1980; Jones, 1975) through rule-learning, even when other strategies might also be available (Fountain et al., 1983).

Although we conclude that our rats differentiated their responses with respect to the two pattern structures in Experiment 2, this conclusion being based on differences in anticipatory repsonding for the 0-pulse quantity, the results cannot be used to determine whether rats anticipated pattern structure prior to experiencing the first elements of a pattern, that is, whether rats could anticipate which pattern would be encountered next. Important in this regard is differential responding on 1 -pulse trials. Rats learned to respond reliably more slowly for the 1-pulse quantity of the monotonic 18-6-1-0 pattern than for larger quantities, as predicted by a rule-learning view. In contrast, the rats never learned to slow their responses in anticipation of the 1-pulse quantity of the nonmonotonic 18-1-6-0 pattern, even after extended training. The latter result may be taken as an indication of the difficulty of the nonmonotonic pattern, and both results are predicted by a rule-learning view of rat serial pattern learning. On the other hand, because the memory of the 18-pulse BSR quantity was the cue predicting both the 6-pulse BSR quantity of the monotonic 18-6-1-0 pattern and 1-pulse quantity of the nonmonotonic 18-1-6-0 pattern, accurate anticipation of the nonmonotonic 1-pulse quantity required recalling some distinguishing feature of the immediately preceding pattern. Because rats did not learn to respond slowly in anticipation of the nonmonotonic 1-pulse quantity, we cannot conclude that rats are able to use such a strategy in this situation. Interestingly, rats were able to use such a strategy with shorter patterns of food reward quantities (Capaldi et al., 1983), suggesting that pattern difficulty may have been the factor most responsible for modulating anticipation of 1-pulse quantities in Experiment 2.

The serial mapping view (Capaldi et al, 1983; Capaldi \& Verry, 1981) of rat serial learning assumes that differential anticipatory responding for 0 -pulse quantities that terminated monotonic and nonmonotonic patterns should be based on anticipation of future rewards - those the rat would receive in the next pattern. This view describes some aspects of rat serial learning in some contexts. However, little evidence was found that anticipation of BSR quantities was modulated by future rewards either in monotonic and nonmonotonic patterns of Experiment 2 or in dispersed monotonic, dispersed nonmonotonic, or embedded patterns of Experiment 3. Although a reliable interaction indicated that rats responded differentially for embedded patterns according to their position within dispersed patterns in Experiment 3 , only the dispersed monotonic group showed a tendency (though not reliable by planned comparisons) to respond more and more slowly in embedded patterns in anticipation of smaller and smaller future quantities. Rats in the dispersed nonmonotonic condition showed almost the opposite effect. In demanding serial learning tasks, then, it may be true that serial mapping will be a small factor in modulating pattern tracking performance, but only when the pattern can be learned through a rule-learning strategy. It is clear that serial mapping played a very small role in modulating rats' learning and performance for the dispersed monotonic and nonmonotonic patterns of Experiment 3. In both Experiment 2 and Experiment 3, however, it should be noted that the serial patterns rats learned were chosen so as to bias the rats to use a rule-learning strategy. Consequently, these patterns could hardly be considered conducive to rats' using other strategies, and it was not surprising that rats failed to do so to any great extent.

\section{Rule-Learning Processes}

Rats appear to learn complex serial patterns using processes similar to those humans use. Rats show a predilection to chunk serial patterns into groups of elements 
that can be easily learned and to encode some representation of rule structure to simplify learning by reducing memory load. These strategies are generally the first that humans adopt in learning patterns similar to those rats encounter in serial learning tasks. Rats and humans, then, appear to share similar approaches to the problem of serial learning - both rats and humans appear to be active processors of information. Rats search patterns for simple formal structure and use cognitive strategies such as chunking and sorting to facilitate locating or encoding simple structure.

Are chunking and sorting processes commonly used by rats? Chunking and sorting processes are most useful to the rat in situations in which patterns are long and well organized, but these processes may also be useful in less demanding serial learning tasks. As shown in Experiment 2 , rats are able to discriminate pattern structures among patterns composed of relatively few elements. In learning more than one kind of pattern or in responding to more than one repetition of the same pattern each day, rats must employ some means of chunking sets of quantities into appropriate patterns, perhaps by using an interpattern interval as a "phrasing cue" (Fountain et al., 1984; Restle, 1972) to facilitate the process. It may be that chunking and sorting are processes commonly used by rats to distinguish between sets of events that are to be stored in a well-organized memory system. In such a system, stimuli encountered in the environment might be remembered according to their temporal order, their contiguity with other similar events, or their relationship to events scattered through time. The important aspect of such a system would be the tendency of the organism to organize day-to-day events into meaningful groups to facilitate learning and, later, memory. Chunking and sorting processes would well serve such a system.

Rats in Experiments 2 and 3 demonstrated the ability to discriminate pattern structures. In Experiment 2, the rats discriminated structures between the two patterns that alternated throughout testing. In Experiment 3, they discriminated structures within patterns. Both results lead to the conclusion that rats' responses to different structures were modulated by characteristics of the pattern, not by a change in pattern-learning strategy. Rats apparently adopted a rule-learning strategy that was either sucessful for learning formally simple patterns or less successful for learning formally complex patterns. Our results suggest that rats bring to the serial learning task a predisposition to use rule-learning along with chunking and sorting processes, and that the characteristics of the pattern modulate the utility of that approach. An appropriate avenue of future research would be to further investigate what features of a pattern determine how the elements of the pattern will be sorted and chunked, and how pattern structure is recognized. One approach might be to investigate the basis of structural ambiguity (Restle, 1976) or irrelevant relations (Hersh, 1974), that is, misleading relationships between pattern elements, and their effects on serial pattern learning.
Knowing how rats recognize structure in serial patterns may lead to a better understanding of how chunking, sorting, and rule-learning processes are related to other learning and memory processes that affect behavior.

\section{REFERENCES}

Bower, G. H., \& Winzenz, D. (1969). Group structure, coding, and memory for serial digits. Journal of Experimental Psychology Monographs, 80(2, Pt. 2), 1-17.

CAPALDI, E. J. (1967). A sequential hypothesis of instrumental learning. In K. W. Spence \& J. T. Spence (Eds.), The psychology of learning and motivation: Advances in research and theory (Vol. 1). New York: Academic Press.

Capaldi, E. J., Molina, P. (1979). Element discriminability as a determinant of serial-pattern learning. Animal Learning \& Behavior, 7, 318-322

Capaldi, E. J.. Nawrocki, T. M., \& Verry, D. R. (1983). The nature of anticipation: An inter-and intraevent process. Animal Learning \& Behavior, 11, 193-198.

CAPAldi, E. J., \& Verry, D. R. (1981). Serial order anticipation learning in rats: Memory for multiple hedonic events and their order. Animal Learning \& Behavior, 9, 441-453.

Fountain, S. B., Evensen, J. C., \& Hulse, S. H. (1983). Formal structure and pattern length in serial pattern learning by rats. Animal Learning \& Behavior, 11, 186-192.

Fountain, S. B., Henne, D. R., \& Hulse, S. H. (1984). Phrasing cues and hierarchical organization in serial pattern learning by rats. Journal of Experimental Psychology: Animal Behavior Processes, 10, 30-45.

Fountain, S. B., \& Hulse, S. H. (1981). Extrapolation of serial stimulus patterns by rats. Animal Learning \& Behavior, 9, 381-384.

Hersh, H. M. (1974). The effects of irrelevant relations on the processing of sequential patterns. Memory \& Cognirion, 2, 771-774.

Hulse. S. H. (1978). Cognitive structure and serial pattern learning by animais. In S. H. Hulse, H. Fowler, \& W. K. Honig (Eds.), Cognitive processes in animal behavior (pp. 311-340). Hillsdale, NJ: Erlbaum.

Hulse, S. H. (1980). The case of the missing rule: Memory for reward vs. formal structure in serial-pattern learning by rats. Animal Learning \& Behavior, 8, 689-690.

Hulse. S. H., \& Campbell, C. E. (1975). "Thinking ahead" in rat discrimination learning. Animal Learning \& Behavior, 3, 305-311.

Hulse, S. H., \& DoRSKY, N. P. (1977). Structural complexity as a determinant of serial pattern learning. Learning and Motivation, 8, 488-506.

Hulse, S. H., \& DORSKY, N. P. (1979). Serial pattern learning by rats: Transfer of a formally defined stimulus relationship and the significance of nonreinforcement. Animal Learning \& Behavior, 7, 211-220.

Hulse, S. H., \& O'LeARY, D. K. (1982). Serial pattern learning: Teaching an alphabet to rats. Journal of Experimental Psychology: Animal Behavior Processes, 8, 260-273.

JoNES, M. R. (1974). Cognitive representations of serial patterns. In B. Kantowitz (Ed.), Human information processing: Tutorials in performance and cognition (pp. 187-229). Hillsdale, NJ: Erlbaum.

JoNES, M. R. (1975). Memory and rule structure in the prediction of serial patterns. Journal of Experimental Psychology: Human Learning and Memory, 104, 295-306.

Miller, G. A. (1956). The magical number seven, plus or minus two: Some limits on our capacity for processing information. Psychological Review, 63, 81-97.

OldS, J. (1956). Neurophysiology of drive. Psychiatric Research Reports, 6, 15-22.

Pribram, K. H., \& Tubbs, W. E. (1967). Short-term memory, parsing, and the primate frontal cortex. Science, 156, 1765-1767.

Restle, F. (1972). Serial patterns: The role of phrasing. Journal of Experimental Psychology, 92, 385-390. 
Restle, F. (1976). Structural ambiguity in serial pattern learning. Cognitive Psychology, 8, 357-381.

Restle, F., \& Burnside, B. L. (1972). Tracking of serial patterns. Journal of Experimental Psychology, 95, 299-307.

Rortblat, H. L. (1982). The meaning of representation in animal memory. Behavioral and Brain Sciences, 5, 353-371.

Roitblat, H. L., Pologe, B., \& Scopatz, R. A. (1983). The representation of items in serial position. Animal Learning \& Behavior, 11, 489-498.

SELF, R., \& GAFFAN, E. A. (1983). An analysis of serial pattern learning by rats. Animal Learning \& Behavior, 11, 10-18.

Simon, H. A. (1974). How big is a chunk? Science, 183, 482-488.
Simon, H. A., \& Kotovsky, K. (1963). Human acquisition of concepts for sequential patterns. Psychological Review, 70, 534-546.

STEIN, L. (1962). An analysis of stimulus-duration preference in selfstimulation of the brain. Journal of Comparative and Physiological Psychology, 55, 405-414.

Tusbs, W. E. (1969). Primate frontal lesions and the temporal structure of behavior. Behavioral Science, 14, 347-356.

(Manuscript received November 9, 1983; revision accepted for publication Feburary 14, 1984.) 